\title{
Low Frequency Active Array Calculations in a Shallow Channel
}

\author{
C. L. Scandrett and D. R. Canright \\ Mathematics Dept., Code MA \\ Naval Postgraduate School \\ Monterey, CA 93943
}

August 10, 2001

\begin{abstract}
The acoustical performance of a submerged linear array of spherical shells in shallow water is examined by combining the T-Matrix method of solving for multiple acoustic interactions among separate bodies, with a model for the ocean as a fluid layer over a half-space of a distinct fluid. The system of source and reflected waves is analyzed by standard approximate contour integration techniques, valid for the far field of the array. Calculations using the results show the effects of shallow water on array performance.
\end{abstract}

\section{INTRODUCTION}

The prediction of the performance of an array of active transducers in a variety of conditions is a significant practical problem, and many methods have been applied to this and related problems.

Here we model active array performance in shallow water by coupling two representations of the acoustic field: a T-matrix representation near the array and a representation of the water "sound channel" with a free surface and a bottom of uniform depth, which overlies a fluid half-space of differing material properties, i.e, the Pekeris model.

The applicability of the Pekeris model has been discussed at length in the memoir of Propagation of Sound in the Ocean by Ewing, Worzel, and Pekeris [1] and has been instrumental in explaining acoustical features of sound propagation in coastal waters. More recently, Tolstoy and Clay [2] have discussed more accurate models for a shallow water channel which include the effects of attenuation in the shallow water "waveguide" by: (a) the introduction of dissipation in the fluid half-space model for the ocean bottom; (b) introduction of a third or multiple fluid layers; or (c) allowance for the transmission of shear waves into the bottom half-space. Extensions of the present work could be applied to any of these more elaborate models with a subsequent increase in the complexity of the problem. For the purpose of demonstrating the effects of coupling between radiating elements in a shallow water array, however, the simplest model (the Pekeris model) has been chosen. 
There have been a series of papers concerning sound propagation in shallow water produced by an array of projectors $[3,4,5,6]$ but in each case, coupling between the individual elements in the array are neglected. In the present work, this coupling is explicitly taken into account by a T-matrix type analysis.

The T-matrix approach accounts explicitly for the mutual interactions (including multiplescattering effects) of elements from the array, as well as the individual radiation of each element caused by an internal driving force. A radiated acoustic field in terms of outgoing spherical harmonic waves from each element is found which is consistent with an array submerged in a fluid of infinite extent. The methodology is similar to that reported by Scandrett and Canright [7] in which source level calculations for arrays of spherical shells in an infinite fluid medium were found.

The method of images yields a superposition of reflected plane waves for each spherical harmonic excited by an element of the array, with the reflection coefficients chosen to give the correct behavior at the free surface and bottom surface. Intermediate steps taken to account for the shallow channel conditions consist of: representing a radiated spherical harmonic in terms of a superposition of plane waves over real and complex angles; constructing an infinite set of images in order to satisfy the free surface and bottom surface boundary conditions; formally summing the series; and evaluating the acoustic far field by an application of contour integration. This sequence of steps has been done in Brekhovskikh [8], and (using a slightly different formulation) by Ewing, Jardetzky, and Press [9], and Pekeris [1], for the case of a monopole point source in a Pekeris model for a shallow water channel.

It should be pointed out that the coupled approach taken in the current work neglects the effects of surface or bottom reflections on the spherical shells themselves. Such effects will be small if the array is located several wavelengths from the surface and bottom, but if such effects were deemed important (perhaps for the case of an array near the free surface of the shallow water channel), the T-matrix approach could easily be extended to include the "free surface image" of near surface array elements.

\section{FORMULATION}

The Pekeris model for a shallow water waveguide is employed in which the upper fluid layer $\left(\rho=1000 \mathrm{~kg} / \mathrm{m}^{3}, c=1524 \mathrm{~m} / \mathrm{s}\right)$ lies over the bottom fluid halfspace $\left(\rho_{1}=2000 \mathrm{~kg} / \mathrm{m}^{3}\right.$, $\left.c_{1}=1600 \mathrm{~m} / \mathrm{s}\right)$. The bottom is assumed to be at a constant depth of $H=10 \mathrm{~m}$. Embedded in the fluid layer is an array of steel spherical thin shells which are forced to oscillate in a breathing mode at a frequency $485 \mathrm{~Hz}(\omega)$. The thin steel shells have a radius of $.5 \mathrm{~m}(\mathrm{a})$, are $.0026162 \mathrm{~m}(h)$ thick and have the material properties : $E=2.07 \times 10^{11} \mathrm{~N} / \mathrm{m}^{2}, \nu=.3$, and $\rho_{s}=7669 \mathrm{~kg} / \mathrm{m}^{3}$.

The governing equations used are:

\section{Fluid:}

$$
\begin{aligned}
& \nabla^{2} p+k^{2} p=0 \quad, \quad k=\frac{\omega}{c} \quad, \quad 0>z>-H \\
& \nabla^{2} p_{1}+k_{1}^{2} p_{1}=0 \quad, \quad k_{1}=\frac{\omega}{c_{1}} \quad, \quad z<-H
\end{aligned}
$$




$$
\begin{gathered}
p(x, y, 0)=0 \quad, \quad p_{1}(x, y, z) \rightarrow 0 \text { as } z \rightarrow-\infty \\
\frac{1}{\rho} \frac{\partial p}{\partial z}(x, y,-H)=\frac{1}{\rho_{1}} \frac{\partial p_{1}}{\partial z}(x, y,-H)
\end{gathered}
$$

Shell:

$$
\begin{gathered}
\Omega^{2} u+u_{\theta \theta}+\cot \theta u_{\theta}+(1+\nu) w_{\theta}-\cot ^{2} \theta u-\nu u \\
-\frac{(3-\nu) \cos \theta}{2 \sin ^{2} \theta} v_{\phi}+\frac{1+\nu}{2 \sin \theta} v_{\theta \phi}+\frac{1-\nu}{2 \sin ^{2} \theta} u_{\phi \phi}=0 \\
\Omega^{2} v+\frac{1-\nu}{2}\left(v_{\theta \theta}+\cot \theta v_{\theta}\right)+\frac{(1-\nu)\left(2-\csc ^{2} \theta\right)}{2} v+\frac{1+\nu}{\sin \theta} w_{\phi} \\
+\frac{(3-\nu) \cos \theta}{2 \sin ^{2} \theta} u_{\phi}+\frac{1}{\sin ^{2} \theta} v_{\phi \phi}+\frac{1+\nu}{2 \sin \theta} u_{\phi \theta}=0 \\
\Omega^{2} w+(1+\nu)\left(u_{\theta}+\cot \theta u+\frac{1}{\sin \theta} v_{\phi}+2 w\right)=-\frac{\left(1-\nu^{2}\right) a^{2}}{E h} p+F(\theta, \phi)
\end{gathered}
$$

\section{Fluid/Structure Interface:}

$$
\nabla p \cdot \vec{n}_{j}=i \omega \rho w_{j}
$$

where

$$
\Omega=\frac{\omega a}{c_{p}} \quad, \quad c_{p}=\sqrt{\frac{E}{\left(1-\nu^{2}\right) \rho_{s}}}
$$

and $\vec{n}_{j}$ is the unit normal to the $j^{\text {th }}$ shell.

A periodic $e^{-i \omega t}$ time dependence is assumed in the solution, and all distances are scaled by the value of the wavenumber $k$, which equals 2 for the values of $\omega$ and $c$ given above, resulting in $k a=1$ for the prescribed problem, with a scaled layer depth of twenty. (See fig. 1 for a schematic of the problem as stated.)

\section{METHODS}

In a previous work of the authors [7], source level calculations of a low-frequency active array of elastic spherical shells with specified internal forcing were found. In that work however, results were applicable only to arrays of projectors in an infinite fluid medium. The acoustic pressure field produced by the array was represented by a superposition of outgoing spherical waves for which far-field source level calculations were made. 
The starting point of the present analysis begins with this series representation of the acoustic field for an unbounded fluid medium.

$$
p(\mathbf{x})=\sum_{l=1}^{L} \sum_{n=0}^{N} \sum_{m=-n}^{n} B_{n m}^{l} h_{n}^{(1)}\left(k r_{l}\right) P_{n}^{m}\left(\cos \theta_{l}\right) e^{i m \phi_{l}}
$$

where the harmonic time dependence $e^{-i \omega t}$ is omitted. The $l$ index refers to individual elements of the array (with the spherical coordinates $\left(r_{l}, \theta_{l}, \phi_{l}\right)$ relative to the position of the $l^{\text {th }}$ element) while the $m, n$ indices are over the spherical harmonics excited by the $l^{\text {th }}$ spherical shell, and whose number is truncated by the value of $N$. The amplitudes $\left(B_{n m}^{l}\right)$ incorporate the forced radiation of the $l^{\text {th }}$ spherical shell, as well as reflection of acoustic pressures from the remaining spherical shells of the array.

The shallow water effects are included by considering a single spherical harmonic excited by a single spherical shell, with a view to determine how the acoustic field produced by this single harmonic must be modified in order to satisfy the free surface and bottom boundary conditions present in the shallow water channel. Summation over all harmonics and all spherical shells can then be done after the effect of a single harmonic of a single array element has been found. The effect of the mutual array element interactions is present by virtue of the manner in which the $B_{n m}^{l}$ have been calculated.

While the radiated field is expressed in terms of spherically spreading waves, the spherical waves must be reformulated in terms of plane waves in order to account for the angle dependence of the reflection coefficient at the interface between two fluid media. Each outgoing spherical wave can be written as a superposition of plane waves as follows $[10,11]$ :

$$
h_{n}^{(1)}(k r) P_{n}^{m}(\cos \theta) e^{i m \phi}=( \pm 1)^{m+n} \frac{i^{-n}}{2 \pi} \int_{0}^{2 \pi} \int_{0}^{\frac{\pi}{2}-i \infty} e^{i k r \cos \gamma} P_{n}^{m}(\cos \alpha) e^{i m \beta} \sin \alpha d \alpha d \beta
$$

where the double integral is over plane waves in all directions (including complex angles, corresponding to "inhomogeneous" plane waves) and $\cos \gamma \equiv \sin \theta \sin \alpha \cos (\phi-\beta) \pm \cos \theta \cos \alpha$ with the upper signs for plane waves propagating above the source $(z>0)$, and the lower sign for plane waves propagating below the source $(z<0)$. (The contour integral in $\alpha$ runs along the real axis to $\pi / 2$, then parallel to the imaginary axis.)

The reflection $\left(V_{1}\right)$ and transmission $(T)$ coefficients for a plane wave of unit amplitude striking a planar interface between two fluids of differing material properties at an angle $\alpha$ with respect to the normal of the interface are given by:

$$
V_{1}(\alpha)=\frac{\delta \cos \alpha-\sqrt{\eta^{2}-\sin ^{2} \alpha}}{\delta \cos \alpha+\sqrt{\eta^{2}-\sin ^{2} \alpha}} \quad T(\alpha)=\frac{2 \cos \alpha}{\delta \cos \alpha+\sqrt{\eta^{2}-\sin ^{2} \alpha}}
$$

where $\delta \equiv \rho_{1} / \rho(\delta=2)$ is the ratio of densities and $\eta \equiv c / c_{1}$ (here $\left.\eta=1524 / 1600\right)$ is the index of refraction. The reflection coefficient at the free surface is $V_{2}=-1$.

Starting with upward and downward traveling plane waves emanating from a source point at an angle of $\alpha$ from vertical, a series of image plane waves are constructed, which when combined with the incident plane waves, produces an infinite series of plane waves satisfying the free surface and interface boundary conditions. Such a series for the region above the 
source point $-z_{0}\left(0>z>-z_{0}\right)$, is found to be:

$$
\begin{aligned}
e^{i\left(k_{x} x+k_{y} y\right)} & \sum_{j=0}^{\infty} V_{1}^{j} V_{2}^{j} e^{i k_{z}\left(z+z_{0}+j 2 H\right)}+(-1)^{m+n} V_{1} e^{i k_{z}\left(z-z_{0}+(j+1) 2 H\right)} \\
& +V_{2} e^{i k_{z}\left(-z+z_{0}+j 2 H\right)}+(-1)^{m+n} V_{1} V_{2} e^{i k_{z}\left(-z-z_{0}+(j+1) 2 H\right)}
\end{aligned}
$$

where $k_{x} \equiv \sin \alpha \cos \beta, k_{y} \equiv \sin \alpha \sin \beta, k_{z} \equiv \cos \alpha$. (Similar series for the regions $-H<z<$ $-z_{0}$ and $z<-H$ can be found.)

Formally summing these series and performing the integration in $\beta$ yields the resultant integral for the acoustic field produced by the $(n, m)$ harmonic:

$$
i^{m-n} e^{i m \phi} \int_{0}^{\pi / 2-i \infty} V(\alpha) P_{n}^{m}(\cos \alpha) J_{m}(k R \sin \alpha) \sin \alpha d \alpha
$$

where $R \equiv \sqrt{x^{2}+y^{2}}$ is the horizontal distance from the source point, and

$$
\begin{aligned}
& V(\alpha)= \\
& \quad \frac{\left(e^{b z}-e^{-b z}\right)\left(e^{b\left(z_{0}-H\right)}+(-1)^{n+m} V_{1}(\alpha) e^{-b\left(z_{0}-H\right)}\right)}{e^{-b h}+V_{1}(\alpha) e^{b H}}, 0>z>-z_{0} \\
& \frac{(-1)^{n+m}\left(e^{-b z_{0}}-e^{b z_{0}}\right)\left(V_{1}(\alpha) e^{b(z+H)}+e^{-b(z+H)}\right)}{e^{-b h}+V_{1}(\alpha) e^{b H}}, \quad-z_{0}>z>-H \\
& \frac{(-1)^{n+m}\left(e^{-b z_{0}}-e^{b z_{0}}\right) T(\alpha) e^{-b_{1}(z+H)}}{e^{-b h}+V_{1}(\alpha) e^{b H}},-H>z
\end{aligned}
$$

with $b=i k \cos \alpha$ and $b_{1}=i k \sqrt{\eta^{2}-\sin ^{2} \alpha}$.

Evaluation of these integrals with slight modifications is detailed in Ewing, Jardetzky, and Press [9]. For the integral valid for $0>z>-z_{0}$, a change of variables produces the integral

$$
I_{0}=\frac{2 i}{k} i^{m-n} e^{i m \phi} \int_{0}^{\infty} \frac{N(u)}{D(u)} J_{m}(R u) P_{n}^{m}\left(\frac{\nu}{k}\right) \sin (z \nu) \frac{u d u}{\nu}
$$

where

$$
\begin{gathered}
N(u)=\left\{\begin{array}{cl}
\delta \nu \cos \left[\left(z_{0}-H\right) \nu\right]+i \nu_{1} \sin \left[\left(z_{0}-H\right) \nu\right] & m+n \text { even } \\
i \delta \nu \sin \left[\left(z_{0}-H\right) \nu\right]+\nu_{1} \cos \left[\left(z_{0}-H\right) \nu\right] & m+n \text { odd }
\end{array}\right. \\
D(u)=\delta \nu \cos [H \nu]-i \nu_{1} \sin [H \nu] \\
\nu=\sqrt{k^{2}-u^{2}} \quad, \quad \nu_{1}=\sqrt{k_{1}^{2}-u^{2}}
\end{gathered}
$$

This integral is evaluated using contour integration, by introducing a small imaginary perturbation of the wavenumbers which shift the poles of the integrand into the first quadrant of the complex $u$ plane. A branch cut following a hyperbolic path emanates from the branch 
point at $u=k_{1}$ and asymptotically approaches the positive imaginary axis (the integrand is "even" in $\nu$ so there is no cut emanating from $u=k$, and the Riemann surface is two-leaved). The Riemann surface chosen is such that the imaginary part of $\nu_{1}$ is positive (to ensure decay of any waves propagating into the bottom half space).

The integral $I_{0}$ is split into two parts (integrals $I_{1}$ and $I_{2}$ ) by rewriting the Bessel function $J_{m}(R u)=\frac{1}{2}\left[H_{m}^{(1)}+H_{m}^{(2)}\right]$ (superscripts of the Hankel functions correspond to subscripts of the I's). The path of integration chosen to determine each of these integrals is shown in fig. 2. The contour integration in the first quadrant correponds to the integral $I_{1}$, while $I_{2}$ 's contour is in the fourth quadrant.

The line integrals $C_{1}$ and $C_{8}$ vanish as $R \rightarrow \infty$. The integrals $C_{4}+C_{9}$ and $C_{5}+C_{10}$ cancel in pairs for the case $m$ is even due to the identity $H_{m}^{(2)}(-R u)=(-1)^{m+1} H_{m}^{(1)}(R u)$. In the event $m$ is odd, the integrals can be shown to decay exponentially as $R \rightarrow \infty$.

The branch cut integrals $\left(C_{2}\right.$ and $\left.C_{3}\right)$ are combined; a change of variables is performed $\left(x^{2}=k_{1}^{2}-u^{2}\right.$, where along $C_{2}, \nu_{1}=-x$ and along $\left.C_{3}, \nu_{1}=x\right)$; and the asymptotic expansion of $H_{m}^{(1)}$ for large argument $(R)$ is used. The combined branch cut integrals can be written:

$$
\int_{0}^{\infty} F(x, \phi, z) e^{i R \sqrt{k_{1}^{2}-x^{2}}} d x \quad \text { as } R \rightarrow \infty
$$

with

$$
\begin{aligned}
F(x, \phi, z) & =\frac{2 i^{-n}}{k} \sqrt{\frac{2}{\pi R i \sqrt{k_{1}^{2}-x^{2}}}} e^{i m \phi} N\left(\sqrt{k_{1}^{2}-x^{2}}\right) P_{n}^{m}\left(\frac{\sqrt{\gamma^{2}-x^{2}}}{k}\right) \\
& \times \frac{\sin \left[z \sqrt{\gamma^{2}-x^{2}}\right]}{\sqrt{\gamma^{2}-x^{2}}} \frac{x^{2} \sin \left[H \sqrt{\gamma^{2}-x^{2}}\right]}{\delta^{2}\left(\gamma^{2}+x^{2}\right) \cos ^{2}\left[H \sqrt{\gamma^{2}-x^{2}}\right]+x^{2} \sin ^{2}\left[H \sqrt{\gamma^{2}-x^{2}}\right]}
\end{aligned}
$$

and $\gamma=\sqrt{k^{2}-k_{1}^{2}}$. Expanding $F(x, \phi, z)$ in a Taylor series about $x=0$, using integration by parts, and a stationary phase analysis results in a leading-order behavior for the branch cut integrals of $O\left(1 / R^{2}\right)$. Therefore in the far-field analysis the branch cut integral is negligible compared to the contribution of the contour integral from the poles.

For the present problem, there are two real roots $\left(u_{p}\right)$ to $D(u)$ which lie in the interval $k_{1}<u_{p}<k$. The two roots are graphically displayed in fig. 3, and have the values: $u_{1} \approx 1.98522$ and $u_{2} \approx 1.93544$. Ultimately we obtain the far-field approximation for the integral $I_{0}$ :

$$
\begin{aligned}
I_{0} & \approx \frac{2 \pi i}{H k} i^{m-n} e^{i m \phi} \\
& \times \sum_{p} \frac{P_{n}^{m}\left(\nu_{p} / k\right) H_{m}^{(1)}\left(R u_{p}\right) \nu_{p} H \sin \left(z \nu_{p}\right) S\left(u_{p}\right)}{H \nu_{p}-\cos \left(H \nu_{p}\right) \sin \left(H \nu_{p}\right)-\delta^{-2} \sin ^{2}\left(H \nu_{p}\right) \tan \left(H \nu_{p}\right)}
\end{aligned}
$$

with $z_{0}$ equal to the depth of the spherical shell's center, $R$ the horizontal distance from the sphere's center, $\nu_{p}=\sqrt{k^{2}-u_{p}^{2}}$, and

$$
S\left(u_{p}\right)= \begin{cases}i \sin \left(z_{0} \nu_{p}\right) & m+n \text { even } \\ \cos \left(z_{0} \nu_{p}\right) & m+n \text { odd }\end{cases}
$$

The residual calculations for the region $-z_{0}>z>-H$ yield identical results, whereas for $z<-H$ a similar calculation produces the result: 


$$
\frac{-2 \pi i}{H k \delta} i^{m-n} e^{i m \phi} \sum_{p} \frac{P_{n}^{m}\left(\nu_{p} / k\right) H_{m}^{(1)}\left(R u_{p}\right) \nu_{p} H \sin \left(H \nu_{p}\right) e^{(z+H)} \sqrt{u_{p}^{2}-k_{1}^{2}} S\left(u_{p}\right)}{H \nu_{p}-\cos \left(H \nu_{p}\right) \sin \left(H \nu_{p}\right)-\delta^{-2} \sin ^{2}\left(H \nu_{p}\right) \tan \left(H \nu_{p}\right)}
$$

Summing now over each spherical harmonic, and over each spherical shell, using the large argument approximation for the Hankel function, and reordering the summations gives

$$
\begin{aligned}
& P \approx \sum_{m=-N}^{N} \sum_{p} A_{m p}\left(\phi_{0}\right) \Psi_{m p}\left(\phi_{0}, z\right) \\
& A_{m p}\left(\phi_{0}\right)=\frac{2 \pi i}{H k} \frac{\nu_{p} H}{\nu_{p} H-\cos \left(\nu_{p} H\right) \sin \left(\nu_{p} H\right)-\delta^{-2} \sin ^{2}\left(\nu_{p} H\right) \tan \left(\nu_{p} H\right)} \\
& \times \sum_{l} e^{-i u_{p}\left(x_{0}^{l} \cos \phi_{0}+y_{0}^{l} \sin \phi_{0}\right)}\left\{\begin{array}{ll}
i \sin \nu_{p} z_{0}^{l} & m+n \text { even } \\
\cos \nu_{p} z_{0}^{l} & m+n \text { odd }
\end{array}\right\} \sum_{n=|m|}^{N} B_{n m}^{l} i^{m-n} P_{n}^{m}\left(\frac{\nu_{p}}{k}\right) \\
& \psi_{m p}\left(\phi_{0}\right)=\sqrt{\frac{2}{\pi R_{0} u_{p}}} e^{i R_{0} u_{p}-i \pi / 4} i^{-m} e^{i m \phi_{0}} \begin{cases}\sin \left(\nu_{p} z\right) & 0>z>-H \\
-\sin \left(H \nu_{p}\right) e^{(z+H) \sqrt{u_{p}^{2}-k_{1}^{2}}} & z<-H\end{cases}
\end{aligned}
$$

with $\left(x_{0}^{l}, y_{0}^{l}, z_{0}^{l}\right)$ the center of the $l^{\text {th }}$ spherical shell, $\phi_{0}$ is the observation angle measured from a reference origin of the array, and $R_{0}$ is a reference far-field distance from the array origin. Note that for a general array, the amplitude has angular dependence, but for a vertical array, values of $x_{0}^{l}$ and $y_{0}^{l}$ are zero, and therefore amplitudes are independent of the observation angle.

In the problem studied here, a vertical array has been used. The "eigenfunctions" for the half space problem (in the $z$ coordinate) are shown in fig. 4. Due to the orthogonality of these eigenfunctions, when determining the power output from the array, the total power output is simply the sum of the power output from each of the eigenmodes. The total power is calculated by performing the integral:

$$
\begin{aligned}
\lim _{R_{0} \rightarrow \infty} & \frac{R_{0}}{2 \omega}\left\{\frac{1}{\rho_{i}} \int_{-\infty}^{-H} \int_{0}^{2 \pi}\left|p\left(R_{0}, \phi, z\right)\right|\left|\frac{\partial p}{\partial R_{0}}\left(R_{0}, \phi, z\right)\right| d \phi d z\right. \\
+ & \left.\frac{1}{\rho} \int_{-H}^{0} \int_{0}^{2 \pi}\left|p\left(R_{0}, \phi, z\right)\right|\left|\frac{\partial p}{\partial R_{0}}\left(R_{0}, \phi, z\right)\right| d \phi d z\right\}
\end{aligned}
$$

The power output per mode for the case of the vertical array is given by:

$$
\Pi_{m p}=\frac{H\left\|A_{m p}\right\|^{2}}{\rho c k \nu_{p} H}\left\{\nu_{p} H-\sin \left(\nu_{p} H\right) \cos \left(\nu_{p} H\right)-\delta^{-2} \sin ^{2}\left(\nu_{p} H\right) \tan \left(\nu_{p} H\right)\right\}
$$

and the total power is then:

$$
\Pi^{\text {total }}=\sum_{m, p} \Pi_{m p}
$$




\section{RESULTS}

As a first test of the resulting formulas, a vertical array of two spherical shells are placed in the middle of the sound channel separated by scaled distances of 6.0 and 12.4 units. For the 6.0 separation, the proportion of power in the first and second modes are .808 and .192 respectively, while for the 12.4 unit separation, the respective powers are 1.0 and 0 . Figs. $5 \mathrm{a}$ and $5 \mathrm{~b}$ plot contour lines of the downfield pressure field as a function of depth and radial distance in meters. As can be seen from fig. 5a (the 6.0 unit separation), a sinusoidally shaped bell (which corresponds to high amplitude or illuminated zones) is present, with a wavelength close to that determined analytically from the formula [2]:

$$
\Lambda_{12}=\frac{2 \pi}{u_{1}-u_{2}} \approx 252 m
$$

Fig. 5b with the 12.4 unit separation is a case in which power is being transmitted in only the first mode. There is no apparent sinusoidal behavior in the field, but one can see the effect of radial drop off by the downward sloping pressure amplitude contour lines.

In the next three figures, power transmission from the two element array are shown for a range of spacing between the spheres. In these figures the shells are centered about the middle of the sound channel. Figs. 6 and 7 graph the proportion of power transmitted into the first and second "modes" of the waveguide. As can be seen, at a separation of about $6 \mathrm{~m}$ all the power is transmitted in the first mode of propagation, while for decreasing separations, amplitude of the second mode increases to its maximum value of about $28 \%$. Also shown in these two figures is the effect of increasing the number of spherical harmonics included in the code. The proportional power calculations are unaffected by an increase in the number of spherical harmonics kept $(N)$ from 2 to 5 .

In addition, figs. 6 and 7 highlight a curious feature of the results. A third set of displayed data shows that the coupling between the spherical shells can be discounted in determining relative power outputs. In this third data set, the radiation field amplitudes $\left(B_{m n}^{l}\right)$ are just the values that a single spherical shell would have in an infinite fluid medium. As one can see, the proportion of power found by neglecting coupling between spherical shells yields nearly identical results to those of the fully coupled case. Only a slight variation results when the shells are in close proximity.

If one considers the total power output from the array, however, a distinct difference between the independently radiating spherical shells, and the coupled shells can be seen in fig. 8. From this figure it is apparent that the coupling between spheres can have a profound effect on the amount of total power delivered by the array - overestimating the total power by as much as $50 \%$ or underestimating it by as much as $20 \%$ when the shells are close. It should be noted that the scaled wavelength of the radiated field is $2 \pi$. Therefore the general conclusion drawn for this simple two element array, is that separations between array elements which are whole number multiples of the wavelength increase, while array separations at alternate half wavelength decrease the total power output, but do not affect the proportion of power distributed between propagating modes of the sound channel.

As a final graph, the effect of adjusting the relative array origin as well as the relative separation of the spherical shells is examined. Fig. 9 is a contour graph of the total power output from the two element array as a function of scaled separation between array elements 
and depth of the array. (A line drawn horizontally across this graph is essentially the graph in fig. 8.) Two "peaks" are apparent. In each of these, the scaled separation is on the order of a wavelength $(\approx 6)$. The peaks are also close to either the free surface or the bottom interface of the fluid layer. This is assumed to be due to the fact that image sources are closer to the actual sources in each case.

\section{CONCLUSIONS}

This technique has the advantage over strictly numerical techniques in that the problem has been decoupled into manageable parts. The methodology could easily be extended to consider actual transducers by the addition of T matrix calculations for the given transducers. Such work is in progress. Using finite elements to determine the T matrix for a given class of transducer, and inputting the matrix into the above semi-analytic technique can be used to determine an arbitrary array configuration's power output at a prescribed frequency of operation.

A limitation of the above analysis is that a relatively simple model of a shallow water environment has been employed. Extensions to more realistic conditions might include using the radiation coefficients found from the coupled elements immersed in an infinite fluid $\left(B_{n m}^{l}\right.$ from above) as inputs to a parabolic approximation method, or a ray method.

\section{ACKNOWLEDGMENTS}

This work is supported in part by the Naval Undersea Warfare Center and by the Antisubmarine Warfare group of the Naval Postgraduate School, Monterey, CA.

\section{REFERENCES}

1. Ewing, W.M., Worzel, J.M., and Pekeris, C.L. (1948). Propagation of Sound in the Ocean (Geol. Soc. Am. Memoir, 27).

2. Tolstoy I. and Clay, C. S. (1987). Ocean Acoustics (American Institute of Physics for the Acoustical Society of America, New York).

3. Buckingham, M. J. (1979). J. Acoust. Soc. Am. 65, 148.

4. Clay, C.S., Wang, Y.Y, and Shang, E.C. (1985). J. Acoust. Soc. Am. 77, 424.

5. Weston, D.E. (1985). J. Sound Vib. 102, 423.

6. Lynch, J.F., Schwartz, D.K., Sivaprasad, K. (1985). J. Acoust. Soc. Am. 78, 575.

7. Scandrett, C. L., and Canright, D. R. (1991). J. Acoust. Soc. Am. 90, 589.

8. Brekhovskikh, L. M. (1980). Waves in Layered Media (Academic Press, New York).

9. Ewing, W.M., Jardetzky, W., and Press, F. (1957). Elastic Waves in Layered Media (McGraw-Hill, New York).

10. Friedman, B., and Russek, J. (1954). Quart. of Appl. Math. 12, 13.

11. Wittmann, R.C. (1988). IEEE Trans. Antennas Prop. 36, 1078. 


\section{FIGURE CAPTIONS}

Figure 1. The Pekeris model of a shallow water channel of uniform depth containing an array of radiating spherical steel shells.

Figure 2. The contour integral evaluated in determining the far field response of the radi-

ating spherical harmonic $h_{m}^{(1)}(k r) e^{i m \phi} P_{n}^{m}(\cos \theta)$. Along $C_{2}$ the real and imaginary parts of $\nu_{1}$ are negative and zero, while along $C_{3} \nu_{1}$ 's real and imaginary parts are positive and zero.

Figure 3. Poles of the Integrand. Intersections of the curves $\tan (\sqrt{H \nu})$ and $\delta \nu / i \nu_{1}$ as functions of $\nu$.

Figure 4. Pressure Field Eigenfunctions. Eigenfunction profiles as functions of depth for the two trapped modes.

Figure 5. Far-Field Pressure Contours for two different spherical shell spacings. For the top figure the array center ias located at the center of the channel, and the spacing between shells is 3 meters. For the bottom figure, the array center is again at the channel center but the separation is now 6.2 meters.

Figure 6. Relative Power Output In First Propagating Mode $\left(\Pi_{01} / \Pi^{\text {total }}\right)$.

Figure 7. Relative Power Output In First Propagating Mode $\left(\Pi_{02} / \Pi^{t o t a l}\right)$.

Figure 8. Total Scaled Power Output (Scaled by the Power Output from a Single Spherical Shell in an Unbounded Fluid)

Figure 9. Total Scaled Power as a Function of Shell Separation and Array Center Depth 